\title{
A INFLUÊNCIA DA TELEVISÃO NOS HÁBITOS, COSTUMES E COMPORTAMENTO ALIMENTAR
}

Cíntia da Conceição Santos ${ }^{1}$, Rosamary Aparecida Garcia Stuchi², Cristina Arreguy-Sena ${ }^{3}$, Nísia Andrade Villela Dessimoni Pinto ${ }^{4}$

RESUMO: Este estudo, observacional e descritivo, teve por objetivo analisar a quantidade e o horário das propagandas veiculadas pela televisão sobre produtos alimentícios, classificar estes de acordo com a pirâmide alimentar e identificar o conteúdo calórico dos mais anunciados por meio das informações nutricionais de seus rótulos. Os dados primários foram obtidos a partir das gravações da programação de duas emissoras brasileiras de televisão aberta, entre julho e dezembro de 2008, nos horários da manhã, tarde e noite, durante 28 dias, e foram tratados segundo frequência e conteúdos pelo sistema de comunicação de Thayer. Identificouse 239 propagandas em 336 horas de gravação, $85 \%$ dos produtos estavam no grupo da pirâmide alimentar, representado por doces e gorduras; observou-se total ausência de frutas e hortaliças. Este estudo aponta a necessidade de empregar estratégias educacionais que promovam adoção de comportamentos alimentares mais saudáveis envolvendo inclusive a mídia televisiva.

PALAVRAS-CHAVE: Televisão; Propaganda; Hábitos alimentares; Estilo de vida.

\section{TELEVISION'S INFLUENCE ON EATING HABITS, PRACTICES AND BEHAVIOR}

ABSTRACT: This observational and descriptive study aimed to analyze the number and the timetabling of television adverts for food products, to classify them according to the Food Guide Pyramid, and to identify the calorie content of the most heavilyadvertised, based on the nutritional information on their packaging. The primary data was collected from recordings of programs from two Brazilian free-view TV companies, from between July and December 2008, from the periods of morning, afternoon and evening, over twenty-eight days, and was analyzed according to frequency and content using Thayer's communication system. 239 adverts were identified in the 336 hours of the recordings. $85 \%$ of the products were in the pyramid group represented by sweets and fats; a total absence of fruits and greens was observed. This study indicates the need for employing educational strategies including the use of television for promoting the adoption of healthier eating habits.

KEYWORDS: Television; Advertising; Eating habits; Lifestyle.

\section{LA INFLUENCIA DE LA TELEVISIÓN EN HÁBITOS, COSTUMBRES Y COMPORTAMIENTO ALIMENTAR}

RESUMEN: Este estudio, observacional y descriptivo, tuvo el objetivo de analizar la cantidad y el horario de las propagandas de productos alimenticios vehiculadas por la televisión, clasificar estes de acuerdo con la pirámide alimentaria así como identificar el contenido calórico de los más anunciados por medio de las informaciones nutricionales de sus rótulos. Los datos primarios fueron obtenidos por grabaciones de la programación de dos emisoras brasileñas de televisión abierta, entre julio y diciembre de 2008, en los horarios de la mañana, tarde y noche, durante 28 días, y fueron tratados según frecuencia y contenidos por el sistema de comunicación de Thayer. Fueron identificadas 239 propagandas en 336 horas de grabación, $85 \%$ de los productos estaban en grupo de la pirámide alimentar representado por dulces y grasas; se observó total ausencia de frutas y hortalizas. Este estudio muestra la necesidad de emplear estrategias educacionales para promover adopción de comportamientos alimentares más saludables involucrando, incluso, la media televisiva

PALABRAS-CLAVE: Televisión; Propaganda; Hábitos alimentares; Estilo de vida.

${ }^{1}$ Enfermeira.

${ }^{2}$ Enfermeira. Doutora em Enfermagem. Professora do Departamento de Enfermagem da Universidade Federal dos Vales do Jequitinhonha e do Mucuri - MG.

${ }^{3}$ Enfermeira. Doutora em Enfermagem. Professora da Faculdade de Enfermagem da Universidade Federal de Juiz de Fora - MG. ${ }^{4}$ Bióloga. Doutora em Ciências dos Alimentos. Professora do Departamento de Nutrição da Universidade Federal dos Vales do Jequitinhonha e do Mucuri - MG. 


\section{INTRODUÇÃO}

O perfil nutricional é compreendido como um dos componentes do processo saúde/doença da população aliado à mudança no perfil epidemiológico das doenças no Brasil, país no qual os eventos infecto-parasitários reduziram e aumentaram as doenças crônicas não transmissíveis, o que remete à preocupação com os hábitos alimentares da população e com os componentes que os lhes influenciam. Há evidências sobre o impacto de comportamentos alimentares sobre o surgimento e/ ou controle de doenças como diabetes, hipertensão arterial, síndrome plurimetabólica, obesidade e eventos cardiovasculares ${ }^{(1-4)}$.

Nas últimas décadas, houve um aumento na prevalência de pessoas obesas em todas as faixas etárias em vários países do mundo, inclusive no Brasil ${ }^{(2-3,5-6)}$. A redução do dispêndio energético, aliada ao aumento de oferta de variedade energética de alimentos, favorece a atual epidemia de obesidade entre crianças, adolescentes e adultos. Atualmente, a principal fonte de lazer envolve atividades em que o uso da atenção e do raciocínio - a exemplo do que ocorre quando são manuseados jogos eletrônicos e assistida televisão ou cinema são priorizados em detrimento de atividades que envolvem o gasto energético pela movimentação corporal. Além disso, a obesidade é uma epidemia global, responsável pela diminuição da expectativa de vida e pelo aumento da mortalidade por doenças cardiovasculares ${ }^{(2-3,5-6)}$.

Paralelamente, o crescimento da violência nos grandes centros urbanos e da carga horária de trabalho dos pais favorecem que crianças e adolescentes tenham como atividade de lazer central assistir televisão (TV). Há relatos que apontam para o fato de que em alguns dias de semana a TV é a companheira das pessoas nos horários das refeições, principalmente nas ocasiões em que os componentes da família, devido a uma imposição de horário ou de ambiente diferenciado de trabalho, ou devido à incapacidade de se reunirem, não conseguem conciliar um horário comum para as refeições, fazendo-as diante dos aparelhos de TV como estratégia de busca de companhia ${ }^{(7-8)}$.

A divulgação de propagandas sobre produtos alimentares de forma atrativa e que os associa a estilos de vida desejados e a jingles - vinhetas de fácil memorização - aliada aos componentes moti- vacionais, às necessidades emocionais/psicológicas e aos desejos individuais ${ }^{(6,9-11)}$ favorecem a explicação da influência dos meios comunicacionais sobre o consumo e escolha dos tipos de alimentos.

Considerando que as pessoas com baixa escolaridade são induzidas pela ideologia capitalista a ampliarem o seu poder de escolha e decisão ${ }^{(12-13)}$, e que o de alimento é fruto de hábitos e costumes influenciados e reforçados por informações que subsidiam a tomada de decisão, a influência da mídia sobre a consolidação de uma orientação alimentar e nutricional da população mostra-se como temática relevante para investigação.

A presente pesquisa teve por objetivo analisar a quantidade e o horário das propagandas veiculadas pela TV sobre produtos alimentícios, classificar estes de acordo com a pirâmide alimentar e identificar o conteúdo calórico dos alimentos mais anunciados, por meio das informações nutricionais presentes nos rótulos. Justifica-se na medida em que possibilita identificar o tipo de influência da mídia sobre comportamento alimentar da população. Isto porque o aumento do tempo de exposição das pessoas ao conteúdo divulgado pela TV pode influenciar suas escolhas e seus hábitos alimentares, contribuindo para adesão a costumes alimentares saudáveis que promovam a saúde, ou para intensificação do sedentarismo e do consumo inadequado de alimentos.

\section{MÉTODO}

Trata-se de estudo observacional e descritivo realizado em dois canais de televisão aberta brasileira. Utilizou-se como critérios para a amostra de conveniência: canais abertos e com as duas maiores audiências no Brasil, denominadas Emissora 1 e Emissora 2. Os dados foram coletados por meio de gravação em vídeo das propagandas, sendo realizada em duas fases: na primeira a coleta ocorreu durante sete dias na Emissora 1 e sete dias na Emissora 2, totalizando 14 dias consecutivos de gravações no mês de julho de 2008 nos seguintes horários: 8:00 às 12:00horas, 14:00 às 18:00 horas e de 19:00 às 23:00 horas. Na segunda fase, que ocorreu no mês de dezembro de 2008, utilizou-se a mesma metodologia acima. Os critérios utilizados para definir o início do período de coleta de dados foi a compatibilidade para a operacionalização da coleta de dados de acordo com as atividades das 
pesquisadoras.

Ao total foram realizadas 12 horas de gravações/dia, durante 28 dias. O instrumento para a coleta de dados foi estruturado em três eixos: 1) frequência da apresentação dos comerciais; 2) qualidade dos alimentos divulgados, segundo a pirâmide alimentar; e 3) meios, instrumentos e estratégias utilizados para atrair a atenção dos consumidores quanto ao dia/hora de exibição, a temática, a emissora, o número de repetições e o tempo de duração das propagandas. Por último houve a verificação das informações nutricionais dos alimentos mais divulgados, por meio da análise dos rótulos dos produtos; para tal foram consultados produtos com as mesmas marcas divulgadas nas propagandas.

A análise do conteúdo das propagandas teve como base o referencial teórico do sistema de comunicação de Thayer ${ }^{(14)}$, ou seja, foi utilizado os conteúdos comunicacionais sobre a forma de divulgação e as características que a tornavam atrativas ou diferencias em relação às outras propagandas. Este sistema foi utilizado como suporte teórico para análise do conteúdo e impacto das propagandas e permitiu analisar os comerciais como oportunidades em que as emissoras veiculam produtos alimentares com determinadas características, que são mensagens capazes de sensibilizar os ouvintes para prestarem mais atenção a elas e, em consequência, incluí-las em seus cardápios a partir de uma estratégia de $m a-$ rketing do produto que está sendo disponibilizado e divulgado pelos patrocinadores.

Segundo este referencial, além de comunicar uma mensagem e seus efeitos, é possível utilizar o ato comunicativo como um processo de interação que é desencadeado a partir da dimensão intrapessoal. Isto equivale dizer que o ato comunicativo é acionado nas ocasiões em que a pessoa receptora de uma mensagem entra em estado de alerta, ou seja, na ocasião em que ela inicia o processo de "levar em conta" o que o outro diz, faz, o que se lê ou o que o outro não diz, não fala, mas deixa implícita em sua mensagem, a qual ocorre na dimensão observacional ou internalizada ${ }^{(14)}$.

Como o objeto de estudo foram os produtos alimentares, e tendo em vista o caráter público dos conteúdos divulgados pelos meios televisivos a presente investigação foi dispensada de ser submetida ao Comitê de Ética.

\section{RESULTADOS}

A intencionalidade de horários para divulgar produtos alimentícios se constitui numa estratégia de marketing cada vez mais usada pelos anunciantes da TV aberta. A predominância do horário de divulgação dos comerciais sobre alimentos, na presente investigação, foi de 8 às 12 horas, perfazendo 127 comerciais apresentados; 72 deles divulgados pela Emissora 1 e 55 pela Emissora 2 (Tabela 1).

Das 239 propagandas alimentícias divulgadas pelas emissoras, e que corresponderam a 336 horas de gravação (Tabela 2), 85\% anunciavam produtos contendo açúcares, óleos e gorduras, ou seja, alimentos localizados no topo da pirâmide alimentar, não sendo identificado nenhum comercial abordando o consumo de frutas e hortaliças (Quadro 1).

Com relação à quantidade de sódio nos alimentos (Tabela 1), observa-se teores elevados, em destaque ao embutido e a batata frita.

Tabela 1 - Teores de sódio (mg/100g) em alguns alimentos de maior frequência veiculados na televisão. Diamantina, 2008

\begin{tabular}{lcc}
\hline \multicolumn{1}{c}{ Alimento } & Rotulo & Tabela TACO * \\
\hline Refrigerante & 12 & 9 \\
Batata frita & 489 & 607 \\
Chocolate & 17 & 9 \\
Leite fermentado & 27 & 33 \\
Embutido & 1230 & 1212 \\
\hline * Tabela de Composição Química de Alimentos (TACO, 2011)
\end{tabular}

Tabela 2 - Quantidade de propagandas alimentícias exibidas, segundo intervalos de horas, em dois canais de televisão. Diamantina, 2008

\begin{tabular}{ccccccc}
\hline Horário de exibição & \multicolumn{2}{c}{ Emissora 1 } & \multicolumn{2}{c}{ Emissora 2 } & \multicolumn{2}{c}{ Total } \\
& $\mathbf{n}$ & $\mathbf{\%}$ & $\mathbf{n}$ & $\mathbf{\%}$ & $\mathbf{n}$ & $\mathbf{\%}$ \\
\hline 08:00 às 12:00 horas & 72 & 60 & 55 & 46,2 & 127 & 53,1 \\
14:00 às 18:00 horas & 28 & 23,3 & 35 & 29,4 & 63 & 26,4 \\
19:00 às 23:00 horas & 20 & 16,7 & 29 & 24,4 & 49 & 20,5 \\
$\quad$ Total & 120 & 100 & 119 & 100 & 239 & 100 \\
\hline
\end{tabular}


Quadro 1 - Frequência de propagandas nas emissoras de televisão segundo a especificação do produto. Diamantina, 2008

\begin{tabular}{|l|c|c|c|c|c|c|}
\hline Conteúdo das propagandas & \multicolumn{3}{|c|}{ Emissora 1 } & \multicolumn{3}{c|}{ Emissora 2 } \\
\hline & $\mathbf{n}$ & $\mathbf{\%}$ & Calorias & $\mathbf{n}$ & $\mathbf{\%}$ & Calorias \\
\hline Chicletes & 37 & 30,8 & $16 \mathrm{kcal}-$ unid & 2 & 1,7 & $16 \mathrm{kcal}$ - unid \\
\hline $\begin{array}{l}\text { Sucos gaseificados e } \\
\text { refrigerantes }\end{array}$ & 13 & 10,8 & $109 \mathrm{kcal} / 1$ itro & 40 & 33,6 & $109 \mathrm{kcal} / 1$ litro \\
\hline Embutidos & 1 & 0,8 & $269 \mathrm{kcal} / 100 \mathrm{mg}$ & 22 & 18,5 & $269 \mathrm{kcal} / 100 \mathrm{mg}$ \\
\hline $\begin{array}{l}\text { Sanduíches e } \\
\text { Batatas fritas }\end{array}$ & $14^{*}$ & $11,7^{*}$ & $\begin{array}{c}491 \mathrm{kcal} / \mathrm{unid} \\
135 \mathrm{kcal} / \mathrm{pct} 45 \mathrm{~g}\end{array}$ & $14^{*}$ & $11,8 *$ & $491 \mathrm{kcal} / \mathrm{unid}$ \\
\hline Sorvete e picolé & 13 & 10,8 & $102 \mathrm{kcal} / 1 \mathrm{~kg}$ & 3 & 2,5 & $135 \mathrm{kcal} / \mathrm{pct} 45 \mathrm{~g}$ \\
\hline Bebida alcoólica & 6 & 5 & $148 \mathrm{kcal} / 1 \mathrm{lata}$ & 5 & 4,2 & $148 \mathrm{kcal} / 1 \mathrm{~kg}$ \\
\hline Leite e fermentados & 8 & 6,7 & $120 \mathrm{kcal} / 200 \mathrm{ml}$ & 12 & 10 & $120 / \mathrm{kcal} / 200 \mathrm{ml}$ \\
\hline Chocolate & 9 & 7,5 & $133 \mathrm{kcal} / 75 \mathrm{~g}$ & 3 & 2,5 & $133 \mathrm{kcal} / 75 \mathrm{~g}$ \\
\hline Café & 10 & 8,3 & $9 \mathrm{kcal} / 100 \mathrm{mg}$ & - & - & \\
\hline Achocolatados conjugados & 4 & 3,4 & $185 \mathrm{kcal} / 200 \mathrm{ml}$ & 5 & 4,2 & $185 \mathrm{kcal} / 200 \mathrm{ml}$ \\
\hline Sucos de frutas & 1 & 0,8 & $120 \mathrm{kcal} / 200 \mathrm{ml}$ & 9 & 7,6 & $120 / \mathrm{kcal} / 200 \mathrm{ml}$ \\
\hline Bolos e panificados & - & - & - & 4 & 3,4 & $134 \mathrm{kcal} / 50 \mathrm{~g}$ \\
\hline Cereais & 2 & 1,7 & $381 \mathrm{cal} / 100 \mathrm{mg}$ & - & - & - \\
\hline Soja e formas processadas & 2 & 1,7 & $459 \mathrm{cal} / 100 \mathrm{mg}$ & - & - & - \\
\hline Total & $\mathbf{1 2 0}$ & $\mathbf{1 0 0}$ & - & $\mathbf{1 1 9}$ & $\mathbf{1 0 0}$ & - \\
\hline
\end{tabular}

* Corresponde a um mesmo comercial.

\section{DISCUSSÃO}

Os resultados deste estudo revelam que a maioria dos produtos alimentícios de propaganda se referem a gorduras e doces, corroborando os resultados de outra pesquisa que demonstrou ser $22,5 \%$ das propagandas exibidas na TV representadas por comerciais alimentícios e que dos 1.395 produtos divulgados, $58 \%$ pertenciam ao grupo da pirâmide alimentar representado por gorduras e doces, com total ausência de propagandas abordando frutas e vegetais ${ }^{(11)}$.

A identificação do conteúdo calórico dos produtos divulgados pelos anúncios, a partir das informações nutricionais presentes nos rótulos, mostrou concentrações de gorduras, carboidratos e sal acima das quantidades recomendadas para uma refeição, fato nem sempre divulgado ou enfatizado pelas propagandas e preconizado pela Organização Mundial de Saúde ${ }^{(2)}$. Segundo esta organização uma dieta humana diária deve conter cerca de $60 \%$ de seu valor energético advinda dos carboidratos e no máximo $30 \%$ dos lipídios (ou gorduras) e 15\% das proteínas.

Cabe destacar ainda, que alimentação hipercalórica ou com altos níveis de sódio, quando associadas ao sedentarismo, vulnerabilizam os consumidores para a ocorrência de problemas cardiovasculares, hipertensão arterial, dislipidemia, diabetes e obesidade ${ }^{(9-10)}$. Há evidências que o aumento destas doenças está vinculado ao estilo de vida da população, caracterizada por vida sedentária e ingestão de alimentos pobres nutricionalmente com perfis calóricos superiores às necessidades orgânicas diárias ${ }^{(15)}$.

As doenças crônicas não transmissíveis são hoje, no Brasil, as maiores causas de morte, sendo que o aumento da obesidade em todo mundo requer a implantação de estratégias que previnam ou reduzam sua incidência e prevalência ${ }^{(15-16)}$.

Considerando a possível influência da mídia televisiva sobre a escolha alimentar dos telespectadores, elaboramos uma refeição conciliando alguns dos produtos divulgados nos comerciais para saber o que poderia causar sobre a saúde dos consumidores. Associamos então: um refrigerante de $700 \mathrm{ml}$, um salgadinho de $45 \mathrm{~g}$, um sanduíche e dois chocolates. Neste caso, o consumidor estaria ingerindo, em apenas um lanche, $73 \%$ (1459 Kcal) de suas necessidades calórica/dia, considerando-se que as calorias recomendadas para um adulto eutrófico é de aproximadamente $2000 \mathrm{Kcal} / \mathrm{dia}^{(17-18)}$ e consumiria uma quantidade de sódio superior à indicada, ou seja, uma quantidade superior a $2.400 \mathrm{mg}$ para que os indivíduos mantenham-se saudáveis, mesmo que não acrescentassem sal para ressaltar o sabor do alimento ${ }^{(19)}$.

A análise do conteúdo calórico dos produtos 
divulgados na TV sugere que a ingestão do lanche combinado com os produtos divulgados causaria vulnerabilidade aos consumidores para desenvolver sobrepeso e hipertensão $\operatorname{arterial}^{(17-18)}$. Se no exemplo anterior houvesse a substituição do refrigerante de $700 \mathrm{ml}$ por um contendo Zero Açúcar, porém com maior teor de sódio, com o mesmo volume, e que possui zero calorias, em apenas um lanche uma pessoa ingeriria $54 \%$ de suas necessidades diária de sódio. Isto equivale dizer que o excesso de sal consumido estaria compensando as calorias, o que do ponto de vista da saúde não é recomendável ${ }^{(18-19)}$. Por outro lado, se houvesse substituição do refrigerante por um suco os produtos alimentares agregariam maior ganho nutricional.

O grupo de alimentos da Pirâmide constituído pelos açúcares e doces, considerados alimentos energéticos e com poucos nutrientes, devem ser consumidos com moderação, sendo recomendados $80 \mathrm{kcal}$, ou seja, duas porções ${ }^{(20)}$.

Na presente investigação, alguns alimentos, como refrigerantes, salgadinhos, lanches, batatas fritas, sorvetes e chocolates, ou seja, alimentos ricos em sódio, estavam presentes nos anúncios nas duas emissoras, fato que retrata divulgação de produtos alimentícios que não são saudáveis às pessoas.

A intencionalidade de horários para divulgar produtos alimentícios sugere um possível direcionamento das propagandas para a população infantil, ou para as mulheres, as quais desenvolvem suas atividades no lar como responsáveis pela lista de compras. Entre os fatores intervenientes sobre o horário de divulgação de determinados comerciais destacamos o interesse em abordar o público infantil, considerando a falta de um padrão alimentar consolidado; a influência afetiva de seus pedidos para os pais e a concentração dos horários de divulgação das $8 \mathrm{~h}$ às $12 \mathrm{~h}$ da manhã. Esses potenciais consumidores, em sua maioria, estudam no horário vespertino e dispõem de maior chance de assistirem televisão em casa pela manhã $\tilde{\tilde{a}}^{(6,8-11)}$.

Outro fator responsável pela fixação das informações divulgadas pela televisão é o tempo de exposição às mensagens. Há relatos de que o tempo médio que as crianças assistem TV é superior a duas horas/dia, ou seja, tempo considerado elevado, tanto para aquelas residentes na área urbana quanto na área rural ${ }^{(20)} \mathrm{e}$ quando esse é superior a quatro horas/dia constitui fator de risco para a criança desenvolver o sobrepeso ${ }^{(6,10-11,20)}$.

Considerando que nos períodos pré-escolar e escolar as crianças passam por mudanças no padrão alimentar, com vistas a construir hábitos e práticas que permanecerão na fase adulta, e que são influenciadas simultaneamente pelos desvios culturais das comidas (representado através de fast-foods, ou seja, refeições rápidas, pelo consumo de enlatados e embutidos), estilos e contextos alimentares (tempo gasto e locais destinados à realização das refeições) e pelo consumo exagerado de alimentos em resposta emocional ou afetiva, é possível redimensionarmos a relevância da participação da família ${ }^{(8,12-13,17)}$ e da escola ${ }^{(9-11)}$ na orientação correta a uma boa nutrição. O período de estudo e o esforço dos familiares em redimensionar o contexto alimentar do estudante é capaz de combater o sedentarismo por meio do incentivo à prática de exercícios físicos e de reduzir o número de horas da criança em frente à $\mathrm{TV}^{(9-11)} \mathrm{e}$, consequentemente, reduzir a exposição delas às mensagens persuasivas de consumo de alimentos excessivamente energéticos e salgados.

Ao analisar o horário de divulgação das propagandas pela televisão e das estratégias de fixação de seus conteúdos a respeito de produtos alimentícios à luz do sistema de comunicação de Thayer ${ }^{(14)}$ foi possível interpretar o marketing das propagandas como dispositivo capaz de sensibilizar os telespectadores a ponto de que "levem em conta" um conteúdo intencionalmente estruturado. A finalidade da mensagem emitida é gerar hábitos e costumes entre os ouvintes que ainda não os possuem formados, e de sensibilizar aqueles que os têm para flexibilizá-los à luz da mensagem divulgada.

As recomendações do uso da pirâmide alimen$\operatorname{tar}^{(17,19-20)}$ baseada no tipo de alimento, na variedade e na quantidade de grupos alimentares (proteínas, carboidratos, vitaminas e sais minerais, óleos e doces, etc.) como roteiro para formação de hábitos, fez surgir uma população de obesos, intensificando a emergência de doenças cardiovasculares ${ }^{(15,17-18)}$. Manter uma alimentação saudável, desde a infância até a fase adulta, constitui numa estratégia de promoção à saúde e de controle dos fatores de risco modificáveis, com impacto na redução de doenças relacionadas à má nutrição, tais como diabetes, hipertensão arterial e dislipidemia, sendo a influência da família e da escola fundamentais na estimulação de comportamentos saudáveis ${ }^{(2,4-5,10,19)}$.

Uma alimentação ideal deve contemplar variedades e proporcionalidades dos grupos alimentares, equilíbrio entre demanda e consumo energético do organismo; quantidades proporcionais às necessidades individuais; intervalos regulares de alimentação; coerência entre alimentação, atividade física e ingesta de água, contextualizando a partir das necessidades energéticas diárias, além de prevenir uma má nutrição (deficiências, 
desequilíbrios e excesso de nutrientes) $)^{6,10-11,17-18)}$.

O esforço persuasivo dos comerciais veiculados pela televisão pode ser constatado pelos altos investimentos publicitários, cujos principais objetivos são os de causar impacto em horários de grande audiência para sua população alvo. Para isto os comerciais reúnem imagens, sons, movimentos e cores para chamar a atenção dos consumidores e desencadear o "levar em conta" de uma necessidade adormecida como forma de influenciar o consumidor em suas escolhas ${ }^{(10-11)}$.

\section{CONSIDERAÇÕES FINAIS}

A presente investigação, ao avaliar a qualidade e a quantidade de propagandas alimentares exibidas na televisão em duas emissoras de televisão nacionais permitiu identificar que os produtos mais calóricos e com maior concentração de sódio do que o recomendado para um adulto saudável são os divulgados.

Quando comparamos a influência de escolas e familiares com a da mídia televisiva é possível identificar que os comerciais possuem uma influência mais duradoura na medida em que utilizam mensagens persuasivas, atraentes e marcantes.

Embora os limites da presente pesquisa seja restrito pelo seu delineamento descritivo, quando consideramos crianças (cujos hábitos alimentares não estão consolidados), adolescentes (cuja flexibilidade de posicionamento é característico desta fase de vida) e adultos (cujos estilos de vida ou necessidade psicoafetivas/emocionais são desgastantes) à luz das recomendações (inter)nacionais para uma alimentação adequada, é possível detectar que a mídia televisiva não tem contribuído para um estilo de vida saudável.

Diante do exposto, a presente investigação aponta a necessidade de empregar estratégias educacionais que promovam adoção de hábitos alimentares mais saudáveis. Ainda, sugere um comprometimento da mídia televisiva com a divulgação de alimentos saudáveis ou a introdução de sinais de alerta para o consumo indiscriminado de produtos que podem causar mal à saúde de forma que ela possa se engajar na consolidação de costumes e comportamentos que promovam a saúde numa concepção ampliada na qual os hábitos alimentares são contemplados.

\section{REFERÊNCIAS}

1. Coutinho JG, Cardoso AJC, Toral N, Silva ACF, Ubarana JÁ, Aquino KKNC, et al. A organização da Vigilância Alimentar e Nutricional no Sistema Único de
Saúde: histórico e desafios atuais. Rev. bras. epidemiol. 2009;12(4):688-99.

2. WHO (SW). The world health report 2003: Shaping the future. Neglected global epidemics: three growing threats. Genebra: World Health Organization; 2003. 204p.

3. Lamounier JA. Transição epidemiológica nutricional em crianças e adolescentes argentinos de áreas carentes. Rev. paul. pediatr. [online] 2009;27(2):124-6.

4. Ribeiro RQ, Lotufo PA, Lamounier JA, Oliveira RG, Soares JF, Botter DA. Fatores adicionais de risco cardiovascular associados ao excesso de peso em crianças e adolescentes. O estudo do coração de Belo Horizonte. Arq. bras. cardiol. 2006;86:406-16.

5. Frutoso MFP, Bismark-Nasr EM, Gambardella AMD. Redução do dispêndio energético e excessivo de peso corporal em adolescentes. Rev. de Nutr. 2003;16(3):257-63.

6. Silva RCR, Malina RM. Sobrepeso, atividade física e tempo de televisão entre adolescentes de Niterói, Rio de Janeiro, Brasil. R. bras. cienc. e mov. 2003; 11(4):63-6.

7. Palma A. Sobrepeso: uma nova realidade no estado nutricional de pré-escolares de Natal, RN: epidemia ou pânico moral? Rev assoc. med. bras [Internet] 2007;54(4). [Acesso em 17 nov 2009] Disponível: http://www.scielo.br/scielo.php?script=sci_ arttext\&pid=S0104-42302008000400009\&lng=en. http://dx.doi.org/10.1590/S0104-42302008000400009

8. Reichembach MT. A refeição em família: um lugar de encontro entre a história da alimentação e da enfermagem. Cogitare enferm. 2004;9(2):53-65.

9. Miotto AC, Oliveira AF. A influência da mídia nos hábitos alimentares de crianças de baixa renda do projeto Nutrir. Rev. paul. pediatria. 2006;24(2):115-20.

10. Crivelaro LP, Sibinelli EC, Ibarra JA, Silva R A publicidade na TV e sua influência na obesidade infantil. UNIrevista. 2006;1(3):1-7.

11. Almeida SS, Nascimento PCBD, Quaioti TCB. Quantidade e qualidade de produtos alimentícios anunciados na televisão brasileira. Rev. saude publica. 2002;36(3):353-5.

12. Santos LAS. Educação alimentar e nutricional no contexto da promoção de práticas alimentares saudáveis. Rev. nutr. Campinas. 2005;18(5):681-92.

13. Canesqui AM, Garcia RWD. Antropologia e nutrição: 
um diálogo possível. Coleção Antrolologia e Saúde. Rio de Janeiro: FIOCRUZ, 2005.

14. Lee Thayer. Comunicação e Teoria da organização In: Dance Frank EX (org). Teoria da comunicação humana. São Paulo: Cultrix; 1973. p. 40-115.

15. Monteiro JBR, Hermsdorff HHM. Atenção nutricional na obesidade e diabetes. Rev. min. educ. fís. Viçosa. 2004;12(2):28-38.

16. Batista MT, Paulino P, Calheiros M. O "Jogo dos Alimentos": Mudança atitudinal face á alimentação e ao sedentarismo em crianças do $10^{\circ}$ Ciclo. Análise psicológ. 2007;2(25):257-69.

17. Mendonça DRB. Alimentação e hábitos saudáveis. In: Gouveia GR, organizadora. Manual de nutrição para profissionais de saúde [online]. São Paulo: Sociedade Brasileira de Diabetes; 2006/2007. p.1-7. [Acesso em 17 nov. 2009]. Disponível: http://www.diabetes.org.br/ livros-e-manuais/550.

18. Ferreira-Sae MC, Gallani MC, Nadruz W, Rodrigues RC, Franchini KG, Cabral PC, Sales ML. Reliability and validity of a semi-quantitative FFQ for sodium intake in low-income and low-literacy Brazilian hypertensive subjects. Public health nutr. 2009; 12(11):2168-73.

19. TACO - Tabela brasileira de composição de alimentos / NEPA - UNICAMP. 4.ed. rev. e ampl. Campinas: NEPA - UNICAMP, 2011. 161p. [Acesso em 17 nov. 2009]. Disponível: <http://www.unicamp.br/nepa/taco/contar/ taco_4_edicao_ampliada_e_revisada.pdf?arquivo=tac.

20. Philippi ST. Pirâmide dos alimentos: fundamentos básicos da nutrição. Barueri, SP: Manole, 2008. 